\title{
Interventional Pain Medicine: A Specialty in the New Millenium
}

\section{Laxmaiah Manchikanti, MD*}

I have not written this letter in some time, and I wanted to take this chance to reflect on the issues of the past year or so before the October annual meeting.

I am honored to serve as the president of the American Society of Interventional Pain Physicians (ASIPP) and assist to move the interventional pain medicine community into the $21^{\text {st }}$ century. ASIPP, formerly known as AOPMA, started with humble beginnings and evolved into the present society representing over 1100 interventional pain practitioners, (a great majority of the interventional pain physicians) from multiple specialties. The achievements of the organization have been beyond my greatest expectations.

- Who could have imagined that interventional pain management would have its own specialty? Interventional pain medicine was not even conceived as a group until three years ago.

- Who would have known that ASIPP would be able to achieve so much for interventional pain medicine and also positively influence the policy makers in Washington in such a short period of time?

- Who would have conceived that a toddler organization, only two and a half years old, with members numbering 850, would visit the Secretary of Health and Human Resources?

- Who would have thought that a 300 member organization in existence for barely a year would convince the Healthcare Financing Administration (HCFA), now the Centers for Medicare and Medicaid (CMS) into adding nine interventional pain medicine codes to the Ambulatory Surgery Center (ASC) approved list, which are not only the bread and butter practice of interventional pain medicine but have also improved access to these services to thousands of pa-

From American Society of Interventional Pain Physicians. *Dr. Manchikanti is the president and executive directorof American Society of Interventional Pain Physicians. Address correspondence: Laxmaiah Manchikanti, MD, 2831 Lone Oak Road, Paducah, Kentucky 42003. E-mail: drm@asipp.org tients in need of these services?

- Who could have dreamed that the Ambulatory Payment Classification (APC) Panel would take an entirely new classification proposed by ASIPP and act on it for reimbursement for hospital outpatient departments (HOPD)?

- Who could have predicted that ASIPP would have a peer-reviewed journal, an enlightening newsletter, and comprehensive texts on the interventional pain management approach to managing low back pain and an extensive guide for documentation billing and coding in interventional pain medicine?

Honestly, none of us dreamed that we could achieve so many things in such a short period of time. Despite these accomplishments, we are faced with even more challenges in the millennium. We always used to worry about regulations for Medicare and Medicaid. Now it appears that we should worry more about new regulations from private insurers. But, if we continue to be persistent, innovative, and creative, (not in billing of course), should realize even greater accomplishments for interventional pain medicine.

It appears that we will have specialty recognition for interventional pain management. Now the question is, when will it be implemented? Once the implementation is carried out (expected to be April or June of next year), we will have the further tasks of enrollment and proving to Medicare that our practice expenses are different from anesthesiology practice expenses (no offense to anyone). That can only be accomplished by the collection of appropriate and reliable data.

The new classification of various interventional techniques presented to the APC Commission is a great start for interventional pain medicine. This classification has already been helpful in increasing reimbursement for hospital outpatient departments. However, it is not simply the reimbursement, but also the recognition by CMS of the complexity of these procedures. It is my hope that CMS will continue to utilize this classification beyond hospital outpatient departments and apply it to ambula- 
tory surgical center, as well as physician payments. However, we need to provide proper data for CMS to base their classifications and improve reimbursements rather than reduce them. On the surface, it may appear that the present classification may reduce reimbursement for ASCs if they follow the same system as HOPD. However, we should realize that this was based on poor quality data. In addition, ASCs function under district rules for themselves, and also distinctly different from HOPDs and physician offices. I would like this classification to be adapted only for purposes of complexity. We also should realize that some of the simple procedures that carry higher reimbursements may lose that. We should, though, always be fair rather than only to advocate for more money. The following is the classification presented by ASIPP, along with the comparison of the proposed classification by CMS which shows some of the changes, and also the proposals not accepted by CMS.

\section{ASIPP PROPOSAL}

\section{Level I}

- Trigger Point, Joint, and Other Injections and Lower Complexity Nerve Blocks

- $\quad$ CPT 20550 (APC 040—\$104.65), 20600 (040), 20605 (040), 20610 (040), 64612 (211-\$164.66), 64613 (211), 64614 (971-\$76.88), 64400-64418 (211), 64425 (211), 64430 (211), 64435 (211), 64445 (211), 64450 (211), 64505 (211), 64508 (211)

- Why clinically homogenous: All single injections on the basis of anatomical landmarks with relatively low incidences of technical complications

- Why resources homogeneous: No fluoroscopy, iv access or fluids, or operating room needed (when only procedure performed); service largely a function of simple alcohol preparation, needle and syringe, local anesthetic and/or steroid; minimal recovery time (5 to 10 minutes)

- Recommendation: Move from APC 211 (and other APCs) to APC 971

\section{CMS PROPOSAL AND MODIFICATIONS}

Level VI nerve injections (proposed as level I interventional techniques)

These codes include:

20550 - trigger point injection

20600 - small joint injection

20605 -intermediate joint injection

20610 - large joint injection

64400 - trigeminal nerve block

64402 - facial nerve block

64405 - greater occipital nerve block

64408 - vagus nerve block

64410 - phrenic nerve block

64412 - spinal accessory nerve block

64413- cervical plexus nerve block

64415 - brachial plexus nerve block

64417 - axillary nerve block

64418 -suprascapular nerve block

64425 -ilioinguinal nerve block

64430 - pudendal nerve block

64435 -paracervical (uterine) nerve block

64445 - sciatic nerve block

64450 - other peripheral nerve or branch block

64505- injection, anesthetic agent; sphenopalatine ganglion 64508 - carotid sinus (separate procedure)

Level III nerve injections (proposed as Level II interventional techniques)

These codes include:

62270 - spinal puncture, lumbar, diagnostic

62272 - spinal puncture, therapeutic, for drainage of spinal fluid (by needle or catheter)

62273 - injection, epidural, of blood or clot patch

62310 - cervical/thoracic epidural

62311 - lumbar/caudal epidural

62318 - continuous epidural - cervical/thoracic

62319 - continuous epidural - lumbar/sacral

64614 - chemodenervation extremity(s) and/or trunk muscle(s) (it may be a misprint. We will comment on this, should be in Level VI)

The reimbursement ranged for these procedures from $\$ 148.79$ to $\$ 180.53$. The new reimbursement would be $\$ 197.27$ with net increase. 


\section{ASIPP PROPOSAL}

\section{Level III}

- Moderate High Complexity: Epidurals, Facet Blocks, and Disk Injections

- 62280-62282 (APC 212), 62290 (No APC), 62291 (No APC), 64420 (211), 64421 (211), 64470 (211), 64472 (211), 64475 (211), 64476 (211), 64479 (211), 64480 (211), 64483 (211), 64484 (211), 64510 (211), 64520 (211), 64530 (211), 64630 (211), 64640 (211)

- Clinically homogenous: precision interventional techniques performed for diagnosis or treatment of conditions involving persistent pain; greater Technical complication risk; more difficult to access relevant sites than Level II procedures

- Resource homogenous: requires fluoroscopy, contrast, sterile environment, sterile preparation, and special spinal or Chiba needles, drugs, local anesthetics, and/or steroids; iv access and fluids; most require iv sedation; tray [sometimes]; moderate to significant recovery time (20 to 45 minutes)

- Recommendation: keep or move to 211 and recalculate with a smaller number of services that bear a tighter resource and clinical relationship to one another

\section{CMS PROPOSAL AND MODIFICATIONS}

Level IV nerve injections (proposed as Level III interventional techniques)

These were moderately high complexity procedures including epidurals, facet blocks and disk injections. These codes include:

62280 - neurolytic subarachnoid

62281 - cervical/thoracic epidural - neurolytic

62282 - lumbar/sacral epidural - neurolytic

64420 - intercostal nerve block - single

64421 - intercostal nerve block - multiple

64470 - facet injection - cervical/thoracic - single

64472 - facet injection - cervical/thoracic - additional

64475 - facet injection - lumbar/sacral- single

64476 - facet injection - lumbar/sacral- additional

64479 - transforaminal cervical/thoracic - single

64480 - transforaminal cervical/thoracic - additional

64483 - transforaminal lumbar/sacral - single

64484 - transforaminal lumbar/sacral - additional

64510 - stellate ganglion block

64520 - lumbar or thoracic sympathetic block

64530 - celiac plexus block

64630 - pudendal nerve neurolysis

64640 - peripheral neurolysis

The reimbursement for these procedures ranged from $\$ 164.66$ to $\$ 180.53$. The reimbursement now will be $\$ 209.98$.

\section{Level V nerve injections (proposed as Level IV interventional} techniques)

These codes include:

62263 - percutaneous epidural adhesiolysis

64600 - neurolytic - trigeminal - small branches

64605 - neurolytic - trigeminal - 2/3 division

64610 - neurolytic - trigeminal - at foramen ovale

64620 - intercostal neurolysis

64622 - facet neurolysis - lumbar/sacral - single

64623 - facet neurolysis - lumbar/sacral - additional

64626 - facet neurolysis - cervical/thoracic - single

64627 - facet neurolysis - cervical/thoracic - additional

64680 - celiac plexus neurolysis

These were reimbursed from $\$ 164.66$ to $\$ 180.53$, which is proposed to be reimbursed at $\$ 387.42$ which is a significant increase. This should facilitate many of the procedures which physicians were unable to offer in hospital settings.
Thus, CMS has accepted most of the recommendations and adjusted the payments mostly in the direction of higher payments for these procedures. CMS also has accepted the request for higher payment for implantables, which 
Table 1. Frequency of utilization of lumbar epidural injections (CPT 62311) by various specialties for 1999 and 1998, in Medicare recipients

\begin{tabular}{|c|c|c|c|c|c|}
\hline Specialty Name & Non-facility & Facility & Total 1999 & Total 1998 & \% change \\
\hline Allergy/Immunology & 0 & 468 & 468 & 121 & $286 \%$ \\
\hline Anesthesiology & 75,761 & 428,313 & 504,074 & 496,611 & $2 \%$ \\
\hline Cardiology & & 65 & 65 & 37 & $76 \%$ \\
\hline Diagnostic radiology & 5,656 & 7,256 & 12,912 & 11,053 & $17 \%$ \\
\hline Emergency medicine & 226 & 755 & 981 & 646 & $52 \%$ \\
\hline Family practice & 1,185 & 1,600 & 2,785 & 2,761 & $1 \%$ \\
\hline Gastroenterology & 36 & 0 & 36 & 30 & $20 \%$ \\
\hline General Practice & 1,422 & 2,178 & 3,600 & 3,175 & $13 \%$ \\
\hline General surgery & 289 & 868 & 1,157 & 1518 & $-24 \%$ \\
\hline Internal medicine & 2,331 & 1,932 & 4,263 & 4,073 & $5 \%$ \\
\hline Interventional radiology & 370 & 1341 & 1,711 & 1,299 & $32 \%$ \\
\hline Nephrology & 21 & 0 & 21 & 84 & $-75 \%$ \\
\hline Neurology & 2,278 & 8,659 & 10,937 & 6,708 & $63 \%$ \\
\hline Neurosurgery & 1,796 & 5,471 & 7,267 & 7,259 & $0 \%$ \\
\hline Nuclear medicine & 0 & 94 & 94 & 51 & $84 \%$ \\
\hline Obstetrics/Gynecology & 16 & 0 & 16 & 28 & $-43 \%$ \\
\hline Ophthalmology & 0 & 18 & 18 & 0 & - \\
\hline Orthopedic surgery & 14,769 & 12,431 & 27,200 & 26,919 & $1 \%$ \\
\hline Osteopathic manipulative therapy & 71 & 291 & 362 & 487 & $-26 \%$ \\
\hline Pathology & 93 & 10 & 103 & 43 & $139 \%$ \\
\hline Pediatric medicine & 23 & 0 & 23 & 0 & - \\
\hline Physical medicine rehabilitation & 9,298 & 21,636 & 30,934 & 23,573 & $31 \%$ \\
\hline Preventive medicine & & 75 & 75 & 74 & $1 \%$ \\
\hline Psychiatry & 300 & 567 & 867 & 533 & $62 \%$ \\
\hline Radiation oncology & 0 & 41 & 41 & 15 & $173 \%$ \\
\hline Rheumatology & 1,353 & 222 & 1,575 & 1,532 & $2 \%$ \\
\hline Thoracic surgery & 145 & 0 & 145 & 164 & $-12 \%$ \\
\hline Urology & 0 & 21 & 21 & 15 & $40 \%$ \\
\hline Others & 4,476 & 3,316 & 7,792 & 9,233 & $-16 \%$ \\
\hline Total & 121,915 & 497,628 & 619,543 & 608,453 & $1.8 \%$ \\
\hline
\end{tabular}

increased morphine implantation from \$554 to \$4824 even though it reduced stimulator implantation payment to $\$ 5,719$ from $\$ 6171$. These payments are in addition to the equipment, hence, this should improve access. It is clear that ASIPP is not only looking out for physicians, but also looking out for facilities to maintain access even though few of the ASIPP members have any interest in hospitals. 
Table 2. Utilization data by with and without Modifier for all services for Medicare recipients for year 1998 and 1999

\begin{tabular}{lccc}
\hline Modifier & $\mathbf{1 9 9 8}$ & $\mathbf{1 9 9 9}$ & \% change \\
\hline 22 & 583037541 & 587275312 & $0.7 \%$ \\
26 & - & 143829 & $2.3 \%$ \\
50 & 94356277 & 96499595 & - \\
51 & & 1678057 & $1.5 \%$ \\
52 & 6468755 & 6566134 & - \\
53 & - & 540530 & - \\
54 & - & 13077 & $4.2 \%$ \\
55 & 323525 & 337272 & $3.3 \%$ \\
56 & 329994 & 340894 & $-16 \%$ \\
62 & 6798 & 5716 & $-2 \%$ \\
66 & 59029 & 57908 & - \\
80 & - & 160 & $-25 \%$ \\
QK & 1129727 & 846195 & $-3.6 \%$ \\
QX & 2434410 & 2346950 & $-96 \%$ \\
QZ & 2420641 & 984 & $-68 \%$ \\
TC & 844756 & 2734 & $7.6 \%$ \\
\hline Total & 5031781 & 5413700 & $0.8 \%$ \\
\hline
\end{tabular}

The next issue is the practice of interventional pain medicine. Once again, I would like to show the statistics of frequency of utilization of various types of interventional procedures for 1999 (the latest year for which statistics are available) and compare them to 1998 statistics which have been published in the past (1). Once again, for purpose of this review, I considered anesthesiologists, physical medicine/rehabilitation physicians, and neurologists who are practicing pain management as interventional pain practitioners. The remaining groups were considered as non-pain practitioners, even though orthopedic surgeons, neurosurgeons, interventional radiologists, and diagnostic radiologists performed a number of interventional pain procedures. One of the drawbacks of these results is that the new interventional pain management codes went into effect starting 1/1/2000, however, CMS has converted this data into 2000 codes for 1998 and 1999.

Table 1 shows the frequency of utilization of lumbar epidural injections utilizing CPT 62311 by various specialties for 1999 and 1998 in only Medicare recipients. This table is designed to show the interventional pain medicine community how lumbar epidural injection is utilized by various specialties. Fortunately, there was only $1.8 \%$ increase in the total procedures with $2 \%$ increase claimed by anesthesiologists, 31\% increase by physical medicine and rehabilitation specialists, and 63\% increase by neurologists. Major decreases were seen in general surgery, nephrology, obstetrics and gynecology, osteopathic manipulative therapy, thoracic surgery and various others. However, there has been an increase also by cardiologists, emergency medicine, physicians, gastroenterologists, psychiatrists, radiation oncologists, and urologists among noninterventional pain practices. The increases of procedures by interventional radiologists was only 32\% compared to $173 \%$ by radiation oncologists and $62 \%$ by psychiatrists and $84 \%$ by nuclear medicine specialists and a whopping $139 \%$ by pathologists. However, in most cases numbers were small.

Table 2 shows utilization data with or without modifier for all services for Medicare recipients for the year 1998 
Table 3. Comparison of frequency of utilization of various types epidural, spinal and disc injection procedures in Medicare recipients for 1999 and 1998, by pain management and other specialties

\begin{tabular}{|c|c|c|c|c|c|c|c|c|c|c|c|}
\hline \multirow[t]{2}{*}{ CPT } & \multicolumn{2}{|c|}{ Anesthesiology } & \multicolumn{2}{|c|}{$\begin{array}{l}\text { Neurology and } \\
\text { Physiatry }\end{array}$} & \multicolumn{2}{|c|}{ Others } & \multicolumn{2}{|c|}{ Total } & \multirow[t]{2}{*}{$\begin{array}{l}\text { Total } \\
1999\end{array}$} & \multirow[t]{2}{*}{$\begin{array}{l}\text { Total } \\
1998\end{array}$} & \multirow[t]{2}{*}{$\begin{array}{l}\text { Percent } \\
\text { change }\end{array}$} \\
\hline & Non-facility & Facility & Non-facility & Facility & Non-facility & Facility & Non-facility & Facility & & & \\
\hline $\begin{array}{l}62263 \text { Epidural } \\
\text { lysis of adhesions }\end{array}$ & 227 & 1005 & 0 & 0 & 78 & 248 & 305 & 1253 & 1558 & 1001 & $56 \%$ \\
\hline $\begin{array}{l}62280 \text { Subarac- } \\
\text { hnoid neurolysis }\end{array}$ & 59 & 130 & 0 & 0 & 16 & 28 & 75 & 158 & 233 & 226 & $3 \%$ \\
\hline $\begin{array}{l}62281 \text { Cervical } \\
\text { epidural neurolysis }\end{array}$ & 315 & 1001 & 62 & 17 & 66 & 108 & 443 & 1126 & 1569 & 1719 & $-9 \%$ \\
\hline $\begin{array}{l}62282 \text { Lumbar } \\
\text { epidural neurolysis }\end{array}$ & 2665 & 4251 & 218 & 54 & 2484 & 1211 & 5367 & 5516 & 10883 & 9543 & $14 \%$ \\
\hline $\begin{array}{l}\text { 62290 Lumbar } \\
\text { discography }\end{array}$ & 433 & 3516 & 53 & 66 & 1372 & 5982 & 1858 & 9564 & 11422 & 8784 & $30 \%$ \\
\hline $\begin{array}{l}62291 \text { Cervical } \\
\text { discography }\end{array}$ & 129 & 391 & 0 & 46 & 151 & 505 & 280 & 942 & 1222 & 1372 & $-11 \%$ \\
\hline $\begin{array}{l}62310 \text { Cervical/ } \\
\text { Thoracic epidural }\end{array}$ & 9493 & 51305 & 637 & 296 & 2939 & 4711 & 13069 & 56312 & 69381 & 64563 & $8 \%$ \\
\hline $\begin{array}{l}62311 \text { Lumbar/ } \\
\text { Sacral epidural }\end{array}$ & 75761 & 428313 & 4759 & 3883 & 41395 & 65432 & 121915 & 497628 & 619543 & 608453 & $2 \%$ \\
\hline $\begin{array}{l}62318 \text { Cervical } \\
\text { continuous epidural }\end{array}$ & 752 & 3519 & 19 & 17 & 167 & 311 & 938 & 3847 & 4785 & 4382 & $9 \%$ \\
\hline $\begin{array}{l}62319 \text { Lumbar } \\
\text { continuous epidural }\end{array}$ & 6054 & 97314 & 20 & 93 & 381 & 4658 & 6455 & 102065 & 108520 & 117440 & $-8 \%$ \\
\hline Total & 95888 & 590745 & 5768 & 4472 & 49049 & 83194 & 150705 & 678411 & 829116 & 817483 & $2 \%$ \\
\hline
\end{tabular}

Table 4. Comparison of frequency of utilization of facet joint injections and neurolytic blocks in Medicare recipients for 1999 and 1998, by pain management and other specialties

\begin{tabular}{|c|c|c|c|c|c|c|c|c|c|c|c|}
\hline \multirow[t]{2}{*}{ CPT } & \multicolumn{2}{|c|}{ Anesthesiology } & \multicolumn{2}{|c|}{$\begin{array}{l}\text { Neurology and } \\
\text { Physiatry }\end{array}$} & \multicolumn{2}{|c|}{ Others } & \multicolumn{2}{|c|}{ Total } & \multirow[t]{2}{*}{$\begin{array}{l}\text { Total } \\
1999\end{array}$} & \multirow[t]{2}{*}{$\begin{array}{l}\text { Total } \\
1998\end{array}$} & \multirow[t]{2}{*}{$\begin{array}{l}\text { Percen } \\
\text { change }\end{array}$} \\
\hline & Non-facility & Facility & Non-facility & Facility & Non-facility & Facility & Non-facility & Facility & & & \\
\hline $\begin{array}{l}64470 \mathrm{C} / \mathrm{T} \text { facet } \\
\text { joint block - single }\end{array}$ & 730 & 3108 & 152 & 72 & 1356 & 1020 & 2238 & 4200 & 6438 & 6286 & $2 \%$ \\
\hline $\begin{array}{l}64472 \mathrm{C} / \mathrm{T} \text { facet } \\
\text { joint block - addit- } \\
\text { ional }\end{array}$ & 86 & 395 & 0 & 0 & 20 & 73 & 106 & 468 & 574 & 349 & $64 \%$ \\
\hline $\begin{array}{l}64475 \text { Lumbar/Sac- } \\
\text { ral facet joint block - } \\
\text { single }\end{array}$ & 9827 & 41298 & 2190 & 1054 & 18855 & 14171 & 30872 & 56523 & 87395 & 84854 & $3 \%$ \\
\hline $\begin{array}{l}64476 \text { Lumbar/Sac- } \\
\text { ral facet joint block } \\
\text { additional }\end{array}$ & 20737 & 90546 & 2271 & 1894 & 21645 & 26077 & 44653 & 118517 & 163170 & 145267 & $12 \%$ \\
\hline $\begin{array}{l}64622 \mathrm{~L} / \mathrm{S} \text { facet } \\
\text { neurolysis - single }\end{array}$ & 1342 & 8743 & 148 & 102 & 1071 & 1673 & 2561 & 10518 & 13079 & 10371 & $26 \%$ \\
\hline $\begin{array}{l}64623 \mathrm{~L} / \mathrm{S} \text { facet } \\
\text { neurolysis - additional }\end{array}$ & 2663 & 22486 & 282 & 218 & 1584 & 3785 & 4529 & 26489 & 31018 & 24255 & $28 \%$ \\
\hline $\begin{array}{l}64626 \mathrm{C} / \mathrm{T} \text { facet } \\
\text { neurolysis - single }\end{array}$ & 0 & 35 & 0 & 0 & 0 & 0 & 0 & 35 & 35 & 25 & $40 \%$ \\
\hline $\begin{array}{l}64627 \mathrm{C} / \mathrm{T} \text { facet } \\
\text { neurolysis - additional }\end{array}$ & 86 & 395 & 0 & 0 & 20 & 73 & 106 & 468 & 574 & 349 & $64 \%$ \\
\hline Total & 35471 & 167006 & 5043 & 3340 & 44551 & 46872 & 85065 & 217218 & 302283 & 271756 & $11 \%$ \\
\hline
\end{tabular}


Table 5. Comparison of frequency of utilization of various types of nerve blocks excluding epidurals, disc injections, and facet joint blocks in Medicare recipients for 1999 and 1998, by pain management and other specialists

\begin{tabular}{|c|c|c|c|c|c|c|c|c|c|c|c|}
\hline \multirow[t]{2}{*}{ CPT } & \multicolumn{2}{|c|}{ Anesthesiology } & \multicolumn{2}{|c|}{$\begin{array}{l}\text { Neurology and } \\
\text { Physiatry }\end{array}$} & \multicolumn{2}{|c|}{ Others } & \multicolumn{2}{|c|}{ Total } & \multirow[t]{2}{*}{$\begin{array}{l}\text { Total } \\
1999\end{array}$} & \multirow[t]{2}{*}{$\begin{array}{l}\text { Total } \\
1998\end{array}$} & \multirow[t]{2}{*}{$\begin{array}{l}\text { Percen } \\
\text { change }\end{array}$} \\
\hline & Non-facility & Facility & Non-facility & Facility & Non-facility & Facility & Non-facility & Facility & & & \\
\hline 64400 Trigeminal N.B. & 800 & 1425 & 491 & 10 & 4204 & 472 & 5495 & 1907 & 7402 & 9363 & $-21 \%$ \\
\hline 64402 Facial N.B. & 234 & 359 & 38 & 0 & 726 & 683 & 998 & 1042 & 2040 & 2523 & $-19 \%$ \\
\hline 64405 Greater occipital N.B. & 4322 & 6972 & 5848 & 329 & 6579 & 776 & 16749 & 8077 & 24826 & 25305 & $-2 \%$ \\
\hline 64408 Vagus N.B. & 0 & 0 & 77 & 0 & 256 & 15 & 333 & 15 & 348 & 258 & $35 \%$ \\
\hline 64410 Phrenic N.B. & 13 & 54 & 0 & 0 & 0 & 0 & 13 & 54 & 67 & 35 & $91 \%$ \\
\hline 64412 Spinal accessory N.B. & 687 & 208 & 87 & 0 & 454 & 0 & 1228 & 208 & 1436 & 1170 & $23 \%$ \\
\hline 64413 Cervical plexus block & 564 & 1713 & 1039 & 23 & 3137 & 487 & 4740 & 2223 & 6963 & 10014 & $-30 \%$ \\
\hline 64415 Brachial plexus block & 1177 & 11614 & 833 & 0 & 1100 & 337 & 3110 & 11951 & 15061 & 9037 & $67 \%$ \\
\hline 64417 Axillary N.B. & 175 & 1256 & 41 & 0 & 278 & 84 & 494 & 1340 & 1834 & 1750 & $5 \%$ \\
\hline 64418 Suprascapular N.B. & 2162 & 1904 & 973 & 0 & 4529 & 137 & 7664 & 2041 & 9705 & 9961 & $-3 \%$ \\
\hline 64420 Intercostal & 831 & 2414 & 120 & 29 & 3387 & 357 & 4338 & 2800 & 7138 & 7936 & $-10 \%$ \\
\hline 64421 Intercostal N. Blocks & 2868 & 9291 & 408 & 15 & 4316 & 1413 & 7592 & 10719 & 18311 & 19485 & $-6 \%$ \\
\hline 64425 Ilio-Inguinal N.B. & 1080 & 2495 & 77 & 0 & 1517 & 386 & 2674 & 2881 & 5555 & 5557 & $-.04 \%$ \\
\hline 64430 Pudendal N.B. & 88 & 93 & 0 & 0 & 272 & 79 & 360 & 172 & 532 & 757 & $-30 \%$ \\
\hline 64435 Paracervical N.B. & 16 & 10 & 0 & 0 & 1339 & 72 & 1355 & 82 & 1437 & 1,926 & $-25 \%$ \\
\hline 64445 Sciatic N.B. & 3096 & 2839 & 1255 & 23 & 11971 & 304 & 16322 & 3166 & 19488 & 22513 & $-13 \%$ \\
\hline 64450 Peripheral N.B. & 12423 & 14633 & 2410 & 89 & 86417 & 6175 & 101250 & 20897 & 122147 & 127904 & $-5 \%$ \\
\hline 64479 C/T Transforaminal epidural - single & 279 & 691 & 361 & 0 & 1559 & 323 & 2199 & 1014 & 3213 & 3292 & $-2 \%$ \\
\hline $\begin{array}{l}64480 \mathrm{C} / \mathrm{T} \text { Transforaminal epidural - each } \\
\text { additional }\end{array}$ & 2027 & 2505 & 1046 & 75 & 6447 & 831 & 9520 & 3411 & 12931 & 17066 & $-24 \%$ \\
\hline 64483 L/S Transforaminal - single & 3797 & 9185 & 5059 & 187 & 21593 & 4930 & 30449 & 14302 & 44751 & 45385 & $-1 \%$ \\
\hline 64484 L/S Transforaminal - each additional & 6095 & 7514 & 3137 & 238 & 19427 & 2605 & 28659 & 10357 & 39016 & 51487 & $-24 \%$ \\
\hline 64505 Sphenopalatine ganglion block & 247 & 308 & 14 & 0 & 5271 & 54 & 5532 & 362 & 5894 & 6532 & $-10 \%$ \\
\hline 64510 Stellate ganglion block & 1723 & 9079 & 165 & 38 & 215 & 406 & 2103 & 9523 & 11626 & 12968 & $-10 \%$ \\
\hline 64520 Lumbar/Thoracic sympathetic block & 1974 & 7571 & 665 & 68 & 2073 & 552 & 4712 & 8191 & 12903 & 14637 & $-12 \%$ \\
\hline 64530 Celiac plexus block & 126 & 1019 & 0 & 0 & 11 & 173 & 137 & 1192 & 1329 & 1538 & $-14 \%$ \\
\hline 64600 Trigeminal neurolysis & 64 & 277 & 42 & 10 & 273 & 106 & 379 & 393 & 772 & 735 & $5 \%$ \\
\hline 64605 Trigeminal neurolysis & 42 & 72 & 0 & 0 & 34 & 43 & 76 & 115 & 191 & 209 & $-9 \%$ \\
\hline 64610 Trigeminal neurolysis & 0 & 264 & 0 & 24 & 0 & 410 & 0 & 698 & 698 & 561 & $24 \%$ \\
\hline $\begin{array}{l}64613 \text { Chemodenervation cervical spinal } \\
\text { muscle }\end{array}$ & 133 & 313 & 7553 & 2033 & 1343 & 613 & 9029 & 2959 & 11988 & 16606 & $-28 \%$ \\
\hline 64620 Intercostal neurolysis & 212 & 1262 & 0 & 0 & 43 & 238 & 255 & 1500 & 1755 & 1752 & $.17 \%$ \\
\hline 64630 Pudendal nerve neurolysis & 0 & 16 & 0 & 0 & 0 & 0 & 0 & 16 & 16 & 16 & $0 \%$ \\
\hline 64640 Peripheral neurolysis & 1075 & 2590 & 2319 & 839 & 10962 & 3148 & 14356 & 6577 & 20933 & 17375 & $20 \%$ \\
\hline 64680 Celiac plexus neurolysis & 107 & 702 & 0 & 0 & 0 & 211 & 107 & 913 & 1020 & 1129 & $-10 \%$ \\
\hline Total & 48437 & 100648 & 34058 & 4030 & 199733 & 26420 & 282228 & 131098 & 413326 & 446782 & $8 \%$ \\
\hline
\end{tabular}

and 1999 with a very slight increase in total services provided to Medicare recipients over a period of one year.

Table 3 shows a comparison of frequency of utilization of various types of epidural, spinal, and disc injection procedures for Medicare recipients for 1998 and 1999 by pain management and other specialists. This showed a 56\% increase in epidural lysis of adhesions with CPT 62263 followed by $30 \%$ increase in lumbar discography and 14\% increase in neurolytic lumbar epidural blocks. There was an $11 \%$ reduction in cervical discography, $9 \%$ reduction in cervical epidural neurolysis, and 8\% reduction in continuous lumbar epidural infusions. Overall, there was a $2 \%$ increase of these procedures in Medicare recipients. 
Table 6. Summary of frequency of utilizations of various categories interventions in Medicare population for 1999 and 1998, by pain management and other specialists

\begin{tabular}{|c|c|c|c|c|c|c|c|c|c|c|c|}
\hline & \multicolumn{2}{|c|}{ Anesthesiology } & \multicolumn{2}{|c|}{$\begin{array}{l}\text { Neurology and } \\
\text { Physiatry }\end{array}$} & \multicolumn{2}{|l|}{ Others } & \multicolumn{2}{|l|}{ Total } & \multirow[t]{2}{*}{$\begin{array}{l}\text { Total } \\
1999\end{array}$} & \multirow[t]{2}{*}{$\begin{array}{l}\text { Total } \\
1998\end{array}$} & \multirow[t]{2}{*}{$\begin{array}{l}\text { Percent } \\
\text { change }\end{array}$} \\
\hline & Non-facility & Facility & Non-facility & Facility & Non-facility & Facility & Non-facility & Facility & & & \\
\hline $\begin{array}{l}\text { Epidural, spinal, and } \\
\text { disk injections }\end{array}$ & 95888 & 590745 & 5768 & 4472 & 49049 & 83194 & 150705 & 678411 & 829116 & 817483 & $2 \%$ \\
\hline Facet joint blocks & 35471 & 167006 & 5043 & 3340 & 44551 & 46872 & 85065 & 217218 & 302283 & 271756 & $11.1 \%$ \\
\hline $\begin{array}{l}\text { Other types of nerve } \\
\text { blocks }\end{array}$ & 48437 & 100648 & 34058 & 4030 & 199733 & 26420 & 282228 & 131098 & 413326 & 446782 & $-8 \%$ \\
\hline Total & 179796 & 858399 & 44869 & 11842 & 293333 & 156486 & 517998 & 1026727 & 1544725 & 1536021 & $1 \%$ \\
\hline
\end{tabular}

Table 4 illustrates the frequency of utilization of facet joint injections and neurolytic blocks. Overall, there was $11 \%$ increase of all facet joint procedures with $64 \%$ increase seen with cervical joint blocks at additional levels, as well as cervical facet joint neurolysis at additional levels, followed by $40 \%$ increase in cervical facet neurolysis for subsequent levels and over $25 \%$ increase for lumbar facet neurolysis.

Table 5 shows statistics of various types of nerve blocks for 1998 and 1999. This table describes many of the somatic and sympathetic and peripheral nerve blocks. Overall, there was an $8 \%$ decrease in the total utilization even though there was a $91 \%$ increase seen with phrenic nerve blocks, $67 \%$ increase was seen with brachial plexus blocks. Table 6 shows the summary of frequency of utilization of various categories of interventions in the Medicare population with an overall increase of $1 \%$.
Table 7 shows the frequency of utilization of procedures which are performed only to a minor extent by interventional pain specialists and includes trigger point injections, joint injections and sacroiliac joint injections. For these procedures, there was an overall decrease of $1 \%$ whereas there was a $2 \%$ increase for large joint injections, an $11 \%$ decrease for small joint injections, $6 \%$ for intermediate joint injections and, finally a $3 \%$ decrease for trigger point injections.

Well, so much for statistics. Now let us discuss the future of our society. Interventional pain specialists have been the first ones to fall through the cracks of various traditional medical societies including the AMA. It has been repeatedly stated that it is impossible to prove or disprove when and how much? We had these societies to protect us all these years. However, practices are becoming more specified and fractionated, thus, our efforts also should be

Table 7. Comparison of frequency of utilization of trigger point injections and intraarticular injections by pain management and other specialties for year 1999 and 1998, in Medicare recipients

\begin{tabular}{|c|c|c|c|c|c|c|c|c|c|c|c|}
\hline \multirow[t]{2}{*}{ CPT } & \multicolumn{2}{|c|}{ Anesthesiology } & \multicolumn{2}{|c|}{$\begin{array}{c}\text { Neurology \& } \\
\text { Physiatry }\end{array}$} & \multicolumn{2}{|c|}{ Others } & \multicolumn{2}{|c|}{ Total } & \multirow[t]{2}{*}{$\begin{array}{l}\text { Total } \\
1999\end{array}$} & \multirow[t]{2}{*}{$\begin{array}{l}\text { Total } \\
1998\end{array}$} & \multirow[t]{2}{*}{$\begin{array}{l}\text { Percen } \\
\text { change }\end{array}$} \\
\hline & Non-facility & Facility & Non-facility & Facility & Non-facility & Facility & Non-facility & Facility & & & \\
\hline $\begin{array}{l}20550 \text { Trigger point } \\
\text { injection }\end{array}$ & 105479 & 93073 & 35747 & 731 & 897306 & 26027 & 1038532 & 119831 & 1158363 & 1188776 & $-3 \%$ \\
\hline $\begin{array}{l}20600 \text { Small joint } \\
\text { injection }\end{array}$ & 785 & 1556 & 377 & 0 & 359125 & 7859 & 360287 & 9415 & 369702 & 414799 & $-11 \%$ \\
\hline $\begin{array}{l}20605 \text { Intermediate } \\
\text { joint injection }\end{array}$ & 2210 & 3360 & 901 & 28 & 419868 & 16353 & 422979 & 19741 & 442720 & 468642 & $-6 \%$ \\
\hline $\begin{array}{l}20610 \text { Large joint } \\
\text { injection }\end{array}$ & 20026 & 32810 & 4370 & 258 & 2357131 & 101707 & 2381527 & 134775 & 2516302 & 2471012 & $2 \%$ \\
\hline $\begin{array}{l}27096 \text { Sacro-iliac } \\
\text { joint injection }\end{array}$ & 58 & 315 & & 0 & 365 & 1543 & 423 & 1858 & 2281 & 2374 & $-4 \%$ \\
\hline Total & 128558 & 131114 & 41395 & 1,017 & 4033795 & 153489 & 4203748 & 285620 & 4489368 & 4545603 & $-1 \%$ \\
\hline
\end{tabular}


specific, focused and fractionated. Many of us belong to various traditional societies such as the American Society of Anesthesiology, the American Academy of Physical Medicine and Rehabilitation, and the American Society of Neurology. In addition, we also belong to various societies representing pain practitioners, if not physicians, including the International Association for the Study of Pain, the American Academy of Pain Medicine, the American Pain Society, and the American Academy of Pain Management. Some of us also belong to the International Spinal Injection Society, the North American Spine Society, the American Academy of Minimally Invasive Surgery, and other traditional societies such as the American Academy of Orthopedic Surgeons and the American Association of Neurological Surgery, etc. While we all agree that we all need to belong to AMA, we are not quite sure what the AMA does for interventional pain physicians. Similarly, we are not sure of specific contributions of any of the above mentioned organizations for interventional pain physicians. Thus, the only way we can survive in the new millennium is with efforts which are focused (even though fractionated), but fulfilling.

Let us look at not only evidence based medicine, but also the evidence of what our parent or traditional societies have done for interventional pain physicians. While I hate not to answer the question, I would like to answer the question of what ASIPP has done for interventional pain medicine. As we prepare to attend our third annual meeting, I would like to say that we are participating in the most prestigious, the most practical and the most well attended interventional pain program anywhere in the United States. However, we should not stop here. We need to form alliances, not only with federal agencies, but with various organizations which are critical in providing and managing patient access to care. We should not become bureaucratic. Our focus must be on the preservation of interventional pain medicine and maintaining access to patient care.
Finally, I would truly like to thank everyone: my family, the staff of the Pain Management Center of Paducah, the executive and other committees, the board of directors, legislators and their staff, and their family. Arent Fox and its staff for helping the interventional pain medicine community realize many of the goals set last September at our annual meeting. Without the hard work and commitment of Bert Fellows, Vidyasagar Pampati; Vijay Singh, MD; David Kloth, MD; Bhupinder Saini, MD; the board; entire membership; and the staff of Arent Fox and the Pain Management Center of Paducah, we never would have been able to accomplish this much in a year. Once again, to every one of the members, non-members, legislators and administrative staff who have put forth an effort to help the American Society of Interventional Pain Physicians to exceed even our own expectations, I thank you wholeheartedly.

I also would like to thank various physicians from the American Society of Anesthesiologists, starting with Neil Swissman, MD, the current president; also Barry Glazer, MD, president elect; James Cottrell, MD, first vice president; Elmer Dunbar, MD, president of the Kentucky Society of Anesthesiologists; Linda Lucas, MD, delegate from Kentucky; Ann Still, MD, pain committee member; Douglas Merrill, MD, chairman of the pain committee; and Alexander Heineberger, MD, member of economics committee. In addition, I would like to thank Samuel Hassenbusch, MD, president of the American Neuromodulation Society; John Oakley, MD, past present of the American Neuromodulation Society; and so many others who have supported us in our endeavors.

\section{REFERENCES}

1. Manchikanti L. State of interventional pain medicine. Pain Physician 2000; 3(3):241-255.

2. Utilization file for 1998-2000 http://www.hcfa.gov/ stats/resource.htm

3. Utilization file for 1999-2001, http://www.hcfa.gov/ stats/resource.htm 